such as their gender and age, experience with VR, professional background, thoughts on the game and recommendations for improvement. In the survey, it was made clear to participants that any information given would be anonymous and personal questions would be limited to their gender, age and profession.

As well as this, participants were instructed to ensure their VR equipment was set up correctly, to stand in line with their motion sensors, ensure their touch controls were securely fastened and to remain stationary throughout the playtest. It was also stated that if any symptom of VR sickness was felt, such as dizziness, headaches, eye soreness, disorientation, etc, they should immediately remove their VR headset.

In the game, participants could engage with:

- A Playroom, where they could get accustomed with the VR controls.

- A picture puzzle, where they needed to put the LMA procedure steps in order.

- A true or false quiz, that tested their academic knowledge on the procedure.

- An operating room, where they could learn/practice the LMA procedure.

$30.8 \%$ of participants were female and $69.2 \%$ were male. Of these individuals, $53.8 \%$ were $20-24$ years old and $46.2 \%$ were 30 - 64 years old. $69.2 \%$ of our participants currently work in the medical field.

The primary feedback from participants was that:

- They enjoyed the game

- The steps for the LMA procedure were accurately conveyed

- Voiced and written instructions needed to be clearer

- Movement and controls should have been smoother

Educational VR video games can be made sufficiently accessible to all age demographics. This can be accomplished by simplifying the VR controls and having variable options to facilitate individual auditory and literacy abilities.

\section{FOREIGN BODY ASPIRATION IN A 13-MONTH OLD BOY - NECESSITY OF RADIOGRAPHIC SCREENING}

${ }^{1}$ Matej Katavić*, ${ }^{1}$ Ivana Trutin, ${ }^{1}$ Ingrid Sitaš, ${ }^{2}$ Maja Bosanac, ${ }^{3}$ Tomislav Baudoin. ${ }^{1}$ Pediatric Department, UHC Sestre Milosrdnice; ${ }^{2}$ Pediatric Department, Children's Hospital Zagreb; ${ }^{3}$ ENT and Head and Neck Surgery Department, UHC Sestre Milosrdnice

\subsection{6/archdischild-2021-europaediatrics.353}

Introduction Foreign body aspiration as one of the leading cause of airway compromise in children remains a serious public health issue. It is estimated that about 3,500 children in the U.S. die each year from foreign body suffocation. Such incidents occur in 60 to as many as $90 \%$ of cases in patients younger than 3 years, more often in male children due to their playfulness and increased curiosity. Detailed medical history, careful clinical status and radiographic examination of the thoracic organs in two directions is cruical for diagnosis, although X-ray is in many cases completely normal. Rigid bronchoscopy is the first choice for making final diagnosis and foreign body extraction. Most foreign bodies can be found in the right main bronchus (30-60\%), left main bronchus (25-50\%), trachea (1-13\%), larynx 3\%, but epidemiological data significantly depend on the authors and institution policy. Foreign body in the respiratory system can cause many complications such as pneumonia, atelectasis, emphysema and bronchiectasis.

Case Report K.K., 13-month boy with inconspicuous perinatal history was hospitalized for RSV positive bronchiolitis when he was 3 months old, and due to impetigo when he was 7 months old. Until the incident he had no other medical issues. At the age of 13 months, he supposedly 'choked' with a dried piece of orange after which an intense cough was noted; the father stated that he became cyanotic, so he vigorously slapped him on the back. The child was initially brought for an examination to the ER of Children's Hospital Zagreb, from where he was reffered to the ER of ENT and Head and Neck Surgery Department for additional opinion and subsequently hospitalized with the aim of further observation. When being brought for a pediatric examination he weighed $12 \mathrm{~kg}$, with no signs of cyanosis and dyspnea, having oxygen saturation of $96 \%$, neat auscultatory finding on the lungs and no asymmetry during percussion. Radiographic imaging revealed shadow measuring $5.5 \mathrm{~cm}$ in length in the projection of the lower part of the trachea and intermediate bronchus (shadow of the hairpin). At that time parents discovered that the child was playing with hairpins that were lying on the floor. Foreign body was successfully removed during rigid bronchoscopy. After the procedure, the child was transferred to the Children's Hospital Zagreb for further care and treatment near the Pediatric Intensive Care Unit. In conclusion, he was discharged without short-term and long-term complications.

Conclusion Aim of this case report is to point out the importance of radiographic imaging during the diagnostic work-up of a patient with suspected foreing body aspiration having neat auscultatory and percussion findings. Prompt intervention and interdisciplinary collaboration are crucial for rapid foreign body extraction, in order to reduce the risk of late complications.

\section{USE OF SOLVENT/DETERGENT - TREATED PLASMA (OCTAPLAS) IN CHILDREN}

Branka Polić*. Pediatric Intensive Care Unit, Department of Pediatrics, University Hospital of Split Spinčicéva 1, Split, Croatia

\subsection{6/archdischild-2021-europaediatrics.354}

Introduction The use of fresh frozen plasma (FFP) has increased significantly in recent years. Clinical practice guidelines recommend plasma transfusion in children and neonates with coagulation disorder and clinically significant bleeding or before invasive procedures with a high risk of bleeding. The use of solvent/detergent (S/D) treatment before freezing significantly reduced the risk of possible side effects. Octaplas is a bio pharmaceutically standardized coagulation active substitute for plasma, and has been used in Europe since 1991. Harmful antibodies and allergic substances have been neutralized and lipid - coated viruses (HIV, HBV and HCV) have been inactivated by S/D. Octaplas contains a well-standardized content of coagulation factors and inhibitors. The structure and function of plasma proteins are not changed, blood cells and leukocyte antibodies, micro vesicles, cell fragments, bioactive lipids are completely removed and the cytokine concentration is lower. Objective To examine the indications for use of Octaplas in children and its efficiency. 